\title{
Photogrammetric Point Clouds Generation in Urban Areas from Integrated Image Matching and Segmentation
}

\author{
Lei $\mathrm{Ye}, \mathrm{Bo} \mathrm{Wu}$ * \\ Department of Land Surveying \& Geo-Informatics, The Hong Kong Polytechnic University, Hung Hom, Kowloon, \\ Hong, Kong - lewis.ye@connect.polyu.hk, bo.wu@polyu.edu.hk
}

\author{
Commission II, WG I
}

KEY WORDS: Photogrammetry, Point Clouds, Urban Areas, Image Matching, Segmentation

\begin{abstract}
:
High-resolution imagery is an attractive option for surveying and mapping applications due to the advantages of high quality imaging, short revisit time, and lower cost. Automated reliable and dense image matching is essential for photogrammetric 3D data derivation. Such matching, in urban areas, however, is extremely difficult, owing to the complexity of urban textures and severe occlusion problems on the images caused by tall buildings. Aimed at exploiting high-resolution imagery for 3D urban modelling applications, this paper presents an integrated image matching and segmentation approach for reliable dense matching of high-resolution imagery in urban areas. The approach is based on the framework of our existing self-adaptive triangulation constrained image matching (SATM), but incorporates three novel aspects to tackle the image matching difficulties in urban areas: 1) occlusion filtering based on image segmentation, 2) segment-adaptive similarity correlation to reduce the similarity ambiguity, 3) improved dense matching propagation to provide more reliable matches in urban areas. Experimental analyses were conducted using aerial images of Vaihingen, Germany and high-resolution satellite images in Hong Kong. The photogrammetric point clouds were generated, from which digital surface models (DSMs) were derived. They were compared with the corresponding airborne laser scanning data and the DSMs generated from the Semi-Global matching (SGM) method. The experimental results show that the proposed approach is able to produce dense and reliable matches comparable to SGM in flat areas, while for densely built-up areas, the proposed method performs better than SGM. The proposed method offers an alternative solution for 3D surface reconstruction in urban areas.
\end{abstract}

\section{INTRODUCTION}

Over the past few decades, urban 3D modelling is emerging as an important problem in photogrammetry and computer vision, with applications in mapping, urban planning, and many other areas. The automatic reconstruction of urban 3D models has become an important research topic since three decades ago (Gruen and Agouris, 1997; Musialski et al., 2013; Zhu et al., 2005).

Airborne LiDAR (Light Detection and Ranging) technology is one of the main techniques for collecting urban 3D data in the past. During the 1990s, the success of approaches based on elevation data was supported by the continuously increasing density and accuracy of point clouds as a result of the fast evolution in LiDAR technology. Subsequently, this technology were improved and direct georeferencing became feasible with sufficient accuracy. The main drawback of LiDAR is that the laser beam samples the earth's surface in fixed pattern, and the flight height of the LiDAR platform limits its application (Brenner, 2005). Despite theoretical considerations and some experiments suggesting high accuracy of the terrain coordinates obtained by means of LiDAR technique, the reality is that the results for large terrain surface often do not meet the expectations (Huising and Pereira, 1998).

Photogrammetry is another widely used technology for obtaining 3D information from photographs (Ackermann and Krzystek, 1991). One of the difficult task in photogrammetry is image matching, which is the process to automatically search for corresponding pixels in stereo pairs, so as to obtain the $3 \mathrm{D}$ object coordinates through photogrammetric space intersection. Suitable image matching algorithms can generate photogrammetric 3D point cloud at an accuracy, reliability and amount of detail, which was only feasible by LiDAR measurement before. This is especially true if high quality images, which means high resolution, good radiometric quality, and high redundancy due to large image overlap, are the source images. High-resolution satellite images at half-meter resolution, such as WorldView-1/2/3/4 and Pleiades-1/2 images, are becoming increasingly available to the earth observation missions (Zhang and Gruen, 2006). The processing of these types of images provides a challenge for algorithmic redesign. This also bring the possibility to reconsider and improve many photogrammetric processing components (Zhang, 2005).

There have been many image matching algorithms developed in the past (Scharstein and Szeliski, 2002). They can be classified into mainly three categories, which are local methods, global methods, and hierarchical methods. The local methods are mainly based on features detected on the images. They are sensitive to the locally ambiguous regions in image. The global methods use elaborate models to describe the matching process. Among them, the Semi-Global Matching (SGM) (Hirschmuller, $2008,2011)$ is the most popular global matching algorithm. SGM aggregates per-pixel matching costs under consideration of smoothness constraints. By minimizing the aggregated cost globally, the disparity map can be finally produce, from which dense matching results can be further derived. Global matching methods have good matching performance in a global scale but they normally require high computational cost. Hierarchical methods combine the local and global methods and take the

\footnotetext{
* Corresponding author.
} 
advantages of both types of methods, such as the self-adaptive triangulation constrained image matching (SATM) (Wu et al., 2011, 2012). However, most of the existing image matching methods are often not well suited to derive the 3D data in urban areas where usually tall buildings are presented, and much human interaction are still required when processing images in urban areas (Habib et al., 2010; Helpke, 1995; Paparoditis et al., 2001; $\mathrm{Wu}$ et al., 2011). This is mainly due to the image matching difficulties. Image matching in urban areas are extremely difficult for two main reasons (Baillard and Maître, 1999; Marinov, 2007):

1) Complexity: the $3 \mathrm{D}$ model of the urban area is very complex, with many height discontinuities and large differences in elevation;

2) Density: the high density of buildings often adjacent to each other, leads to many occlusion and shadows.

On the other hand, the complexity of the textures in urban areas endows the accuracy and convenience of feature based matching, and high density of buildings offers the possibility for more precise edge-based image segmentation. These are the potential advantages of images in urban areas comparing with flat and smooth areas. In the past, there are some research works using segmentation patches to filter or interpolate the disparities for improved image matching (Bleyer and Gelautz, 2005; Bleyer et al., 2011a; Bleyer et al., 2011b; Klaus et al., 2006; Xu et al., 2015; Zhang et al., 2015). The used disparity maps in these methods are relatively smooth due to the interpolation of disparity and the relatively smooth object scene. However, in urban areas the disparities may vary drastically in local areas because of the densely located buildings. Even within the coverage of a single building, the disparity is not guaranteed to be smooth. Therefore, this research integrates image matching together with image segmentation in a dynamic process, in which the triangulationbased matching constraint and segment-based disparity map are both dynamically refined along with the matching propagation process, so that to provide enhanced matching constraints and guidance, and finally to generate dense and reliable photogrammetric 3D point clouds from high-resolution images in urban areas.

The new integrated image matching and segmentation method for photogrammetric point clouds generation in urban areas is presented in Section 2. In Section 3, two different experiments representing different urban types are conducted and results are compared with the point clouds acquired from the airborne laser scanning and the results generated from the SGM matching method. And finally, concluding remarks are presented and discussed in Section 4.

\section{INTEGRATED IMAGE MATCHING AND SEGMENTATION FOR PHOTOGRAMMETRIC POINT CLOUDS GENERATION IN URBAN AREAS}

\subsection{Overview of the Approach}

The integrated image matching and segmentation (Fig. 1) mainly contains four parts, which are image segmentation, feature based matching, occlusion filtering, and dense matching propagation. For a pair of stereo images, feature-based matching will be carried out based on the self-adaptive triangulation constraint The feature based matching contains two parts, feature-to-feature matching and feature-to-area matching. Feature-to-feature matching matches the features directly based on similarity correlation. Firstly, several seed points and edges are obtained on the stereo images, and they are used to construct a pair of initial edge-constrained triangulations on the images. Then, points and edges are matched based on the triangle constraint and other constraints. Feature-to-area matching searches for matches of the remaining features in a reference image that are not successfully matched in the previous feature-to-feature matching step, within a local area in another image constrained by the triangulations (Wu et al., 2012). The newly matched point and edges are inserted into the triangulations and the constrained triangulations are updated dynamically along with the matching propagation. The final results will be the final edge-constrained triangulations generated from the successfully matched points and edges. Details about the self-adaptive triangulation constrained image matching can be found in (Wu et al., 2011, 2012; Zhu et al., 2010).

Image segmentation will be implemented at the beginning of image matching. After that, the initial disparity map will be generated based on the corresponding triangulations, matched features and image segments. The disparity map and the segmentation results will be used to fit the depth planes. Meanwhile, the matching propagates by investigating whether the candidate match can fulfill the model of the depth planes. If it can fulfill the requirement, the candidate match will be accepted as the new match and inserted into the triangulations and the corresponding depth plane will also be updated and densified simultaneously. It should be noted that the occlusion filtering provides a loose constraint on the earlier image matching propagation stage when the disparity map was less accurate due to the smaller amount of matched points. Constraints become tighter as the matched results become more densely distributed during the image matching progress.

In addition to occlusion filtering, a segment-adaptive similarity correlation is employed to reduce the matching ambiguity in the regions close to the building boundaries. And the existing dense matching propagation is improved for better performance of image matching in urban areas.

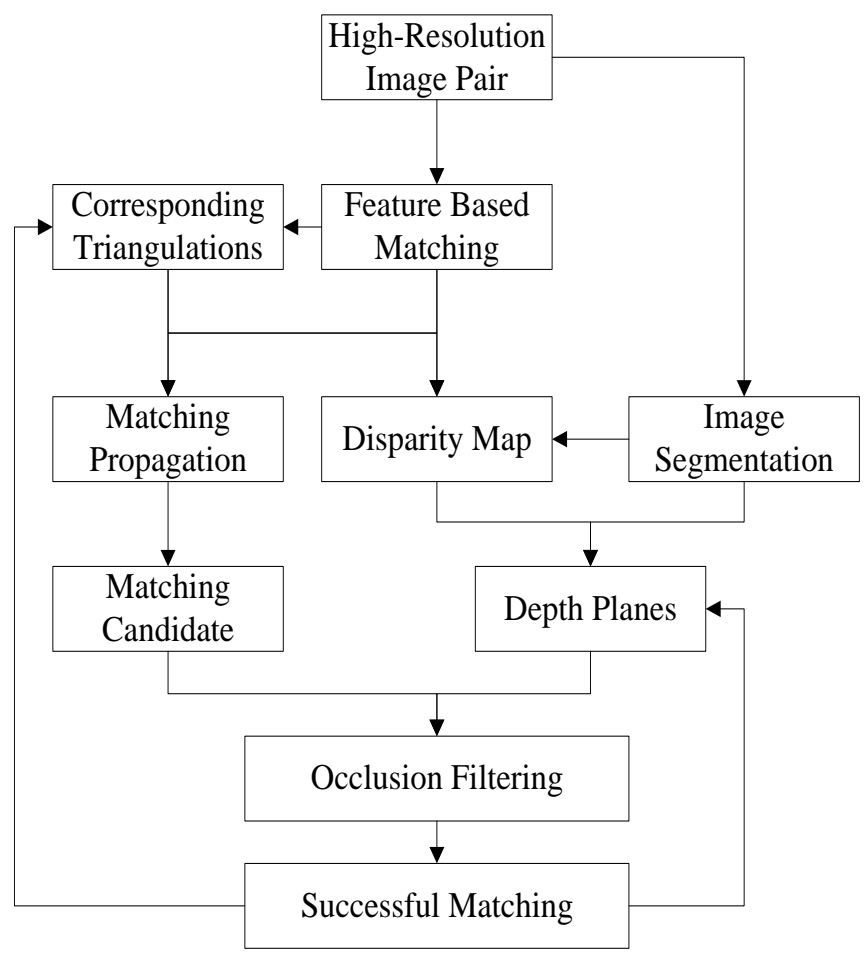

Fig.1 The flowchart of the proposed approach. 


\subsection{Image Segmentation}

Image segmentation is an important step of the proposed approach. This research firstly employs the well-known edge detection method to detect edges on the images. According to experimental analysis, the edge detection method, EDSION (Meer and Georgescu, 2001) (edge detection with embedded confidence), performs more stable and accurate than other methods in high-resolution remote sensing image. In this method, an independent measure of confidence is estimated in the presence of the employed edge model. The EDSION contains three steps: gradient estimation, non-maximum suppression and hysteresis thresholding. It is particularly effective in detecting weak edges without introducing spurious edges (Li et al., 2010).

The extracted edges are also used in the aforementioned edge matching process. Based on the edge matching results, the detected edge in one image can be extended to its full length of the edge length detected on the other image. After that, a local reweight strategy is used to detect the local maximum near the end point of the obtained edges until the edge is intersected with the neighborhood edges. Finally, the closed edges are obtained, and segments are formed through region growing. To make sure the reliability of the segmentation, manual interaction can be further carried out as a supplementary step. Fig. 2 is an example of the segmentation results from this method.

The segmentation results will be used in the next steps of similarity correlation and occlusion filtering.

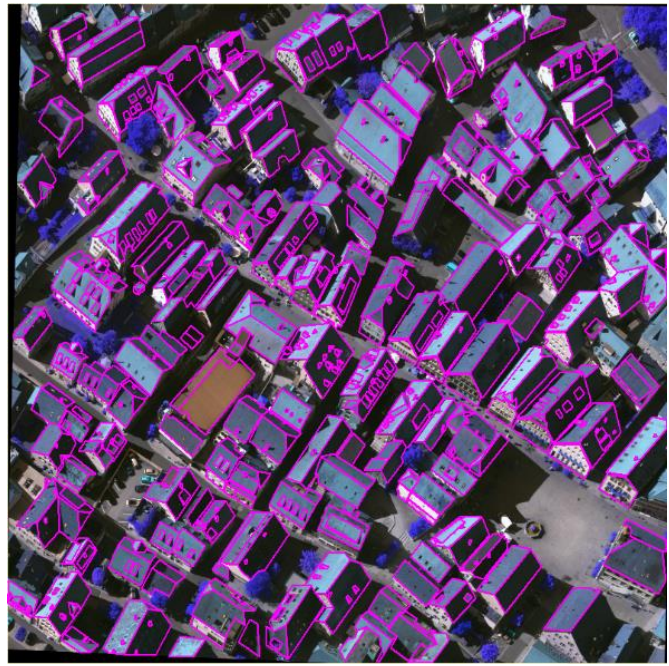

Fig.2 An example of the segmentation results.

\subsection{Segment-Adaptive Similarity Correlation}

The matching cost is exploited for measuring the relation or similarity of the corresponding pixels. However, correlation assumes that the depth is equal for all pixels within the correlation window. This assumption is violated at depth discontinuities (Hirschmüller, 2001).

Fig.3 illustrates the typical decision conflict of the correlation at object border. It is necessary to split the correlation window into two parts to understand the correlation problems. This type of decision conflict is named as border defect. The two parts $a, b$ corresponds to two correlation $C C_{a}$ and $C C_{b}$. It is easy to confirm that the part $a$ owns the higher correlation coefficient (larger area). However, due to the mis-corresponding of the part $b$, the similarity of these part $b$ have a lower similarity. As a whole, the overall correlation may not exceed the predefined threshold, which may lead to the missing of match within this area near the border.

The basic reason for the above problems is the discontinuities within the correlation window. Therefore, this paper proposes a segment-adaptive correlation method for point and line feature features. Fig.4 illustrates the basic idea of the segment-adaptive similarity correlation for points. The gray area means the object (ground), while the white means the ground (object). The line between the gray and white marks the object (e.g., buildings) border. The pixel of interest locates in the middle of the correlation window, in the gray area. According to the segmentation, the interest pixel should have geometric relation with the pixels in gray color. The white area would lead to a decrease of correlation coefficient and mismatching of the interest pixel (or missing of correct matches). Therefore, in this case, the white area will be dismissed when calculating the correlation coefficient

There is a slightly difference while calculating the correlation coefficient of the edge features. In case of the occlusion, if the interest pixel is on the border, the effective correlation area should be the higher part. According to the relationships between the depth (D), the focal length (f), the baseline length (B), and the disparity (d) (normal case photography, which can be extended to other case):

$$
\mathrm{D}=\frac{f}{d} B
$$

The larger disparity means smaller depth. So both part of the correlation are calculated, and the larger disparity wins the matching.

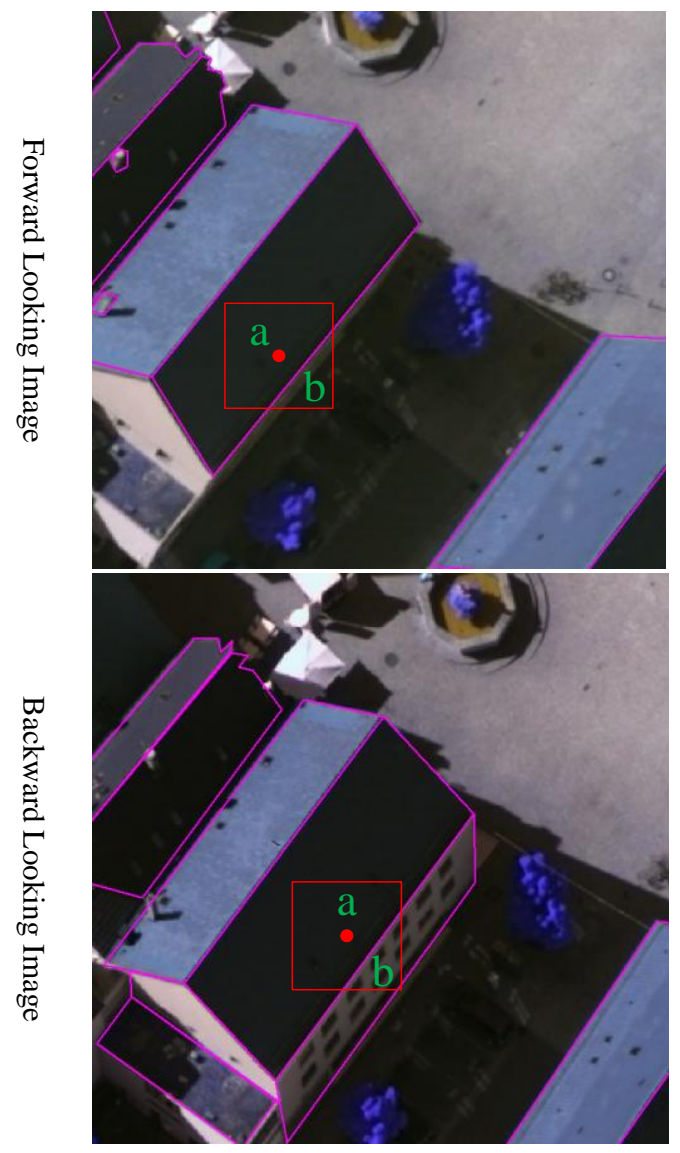

Fig.3 The typical decision conflict of the correlation 


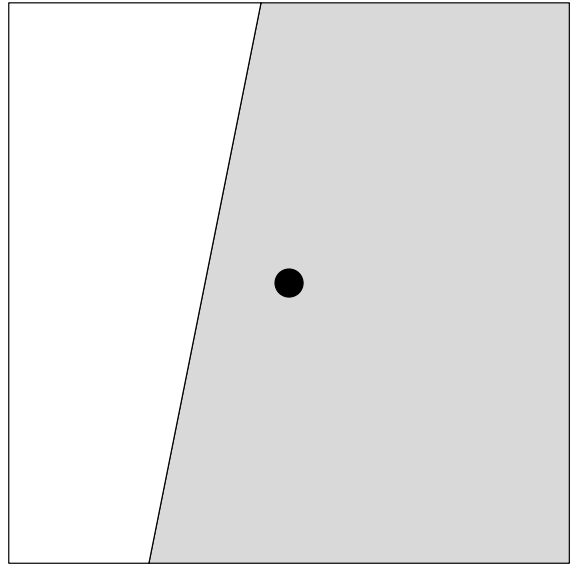

Fig.4 Segment-adaptive similarity correlation

\subsection{Occlusion Filtering}

Occlusion problems are severe in urban areas due to the densely built-up buildings. To reduce the ambiguities caused by occlusion problems in images of urban areas, this research develops an occlusion filter and incorporates it in the image match framework based on segmentation. Fig.5 shows the flowchart of the occlusion filtering.

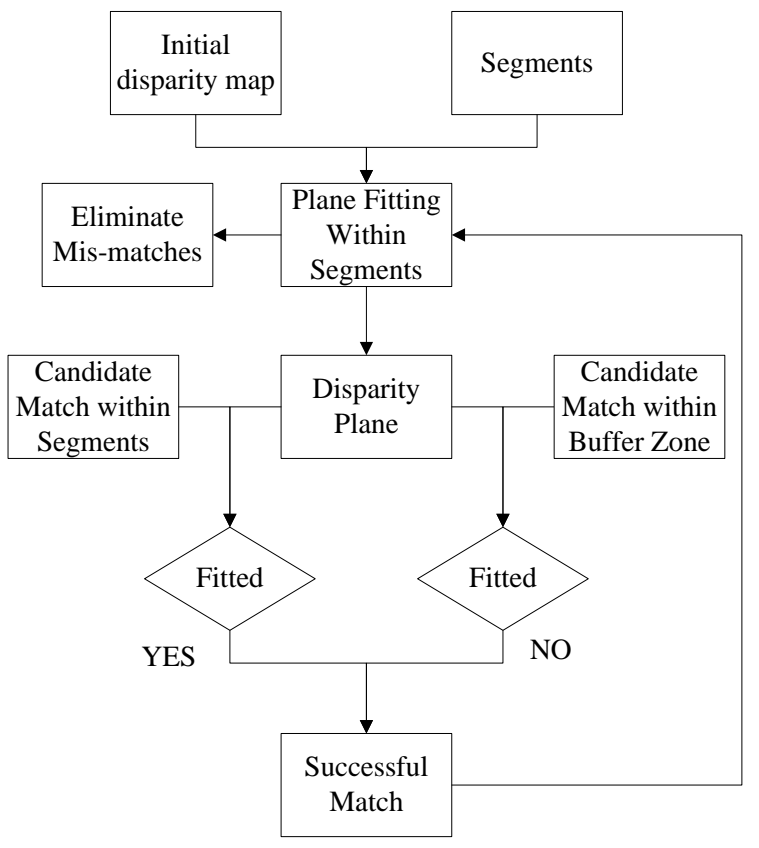

Fig.5 The flowchart of the occlusion filtering.

In Fig. 5, The initial disparity map is generated based on the feature-based matching results (the corresponding triangulations), and the initial segmentation results obtained from previous step. Within each segment, the RANSAC algorithm (Fischler and Bolles, 1981) is used to fit the disparities with a plane model or a quadric surface model robustly. After the surface model is established, the candidate match within the corresponding segment will be checked to see if the disparity can fit the surface model or not. If yes, the candidate match will be accepted as a successful match. For the candidate match within a buffer zone of the segment (within a buffer range outside the segment), the disparity will also be checked, if the disparity can still fit for the surface model, this candidate match must be a wrong match and will not be accepted, otherwise, it will also be categorized as successful match. The newly matched pixels (disparities) will be used to re-fit the surface by RANSAC dynamically, so that the surface will be close to the true surface gradually.

\subsection{Dense Matching Propagation}

After the previous matching, there are still many unmatched pixels. The need for dense matching propagation is still immense. The dense matching propagation contains two strategies, which are matching propagation and disparity interpolation.

The basic principle of the matching propagation is that the disparity within a small region (e.g. 2 pixels) is almost the same. The dense matching uses a matching propagation strategy inspired by the popular dense matching methods (Lhuillier and Quan, 2002; Megyesi and Chetverikov, 2004; Otto and Chau, 1989; Zhang and Gruen, 2006), which is a "best-first" strategy. In this method, the best means the most reliable seeds to guide the matching.

The basic principle of the disparity interpolation is that the disparity of a pixel is correlated with the surrounding pixels. However, this correlation is not simply averaging, which will only be correct when the terrain is smooth. Given a search area around the unmatched pixels, the disparities of matched pixels may be clustered into different clusters, the unmatched pixels must correspond to one cluster among these. Based on this, the disparity interpolation is implemented as follows:

1. Disparity classification: For each unmatched pixel, search for the matched pixels within the surrounding area, and carry out classification of the disparities.

2. Analysis the statistical feature of the disparity cluster, get the mean value and standard deviation of the cluster.

3. Use the mean value and standard deviation of the cluster as the disparity candidate to search for the possible correct match.

The final densely matched results will be used to generate the 3D point clouds through photogrammetric space intersection, and DSMs will be interpolated from the 3D point clouds.

\section{EXPERIMENTAL ANALYSIS}

In order to evaluate the proposed approach, two experiments were carried out and analysed by comparing the results from the proposed approach, the SGM method, and the airborne laser scanning data, based on the accuracy and point density. The evaluation procedure begins by shift the $\mathrm{X}, \mathrm{Y}, \mathrm{Z}$ coordinates to the reference DSM generated from the LiDAR point clouds, to ensure that the evaluation is not biased by errors in the orientation of the images. After shifting, the signed euclidean distance $d$ between the reference DSM and those from the proposed algorithm and SGM are computed. Three statistical indexes, standard deviation, RMSE and maximum error are used to measure the errors.

\subsection{Aerial Images in Vaihingen, Germany}

The test data set from the ISPRS 3D Building Reconstruction Benchmark was used as the first test dataset in this paper. This test data set was captured over Vaihingen in Germany (refer to Fig. 2). The ground resolution of the image is $8 \mathrm{~cm} /$ pixel. The reference dataset used in this experiment is a $3 \mathrm{D}$ point clouds generated from an airborne laser scanner, and the point density varies between 4 and $7 \mathrm{pts} / \mathrm{m}^{2}$. 
The results of the statistical evaluation are shown in Table 1. According to Table 1, the RMSE, the standard deviations and maximum error for the proposed approach are slightly superior than those from the SGM method.

Table 1 The statistical result of the experiment in Vaihingen

\begin{tabular}{|c|c|c|c|c|}
\hline & $\begin{array}{c}\text { RMSE } \\
(\mathrm{m})\end{array}$ & $\begin{array}{c}\text { StD } \\
(\mathrm{m})\end{array}$ & $\begin{array}{c}\text { Maximum } \\
(\mathrm{m})\end{array}$ & $\begin{array}{c}\text { Density } \\
\left(\mathrm{pts} / \mathrm{m}^{2}\right)\end{array}$ \\
\hline $\begin{array}{c}\text { Proposed } \\
\text { Method }\end{array}$ & 1.57 & 1.56 & 7.39 & 106.7 \\
\hline SGM & 2.10 & 2.10 & 9.11 & 101.0 \\
\hline
\end{tabular}

The shaded DSM derived from the proposed approach, the SGM and LiDAR (in order) are shown from Fig.6 to Fig.8. It appears that the DSM from the proposed approach shows more sharping building boundaries compared with those from the SGM method. And in flat regions the DSM from the proposed approach is more smooth than those from the SGM method. The vertical walls near the main streets from the proposed approach is closer to the LiDAR data. This is exactly attributed to the segmentation-based occlusion filtering and similarity correlation.

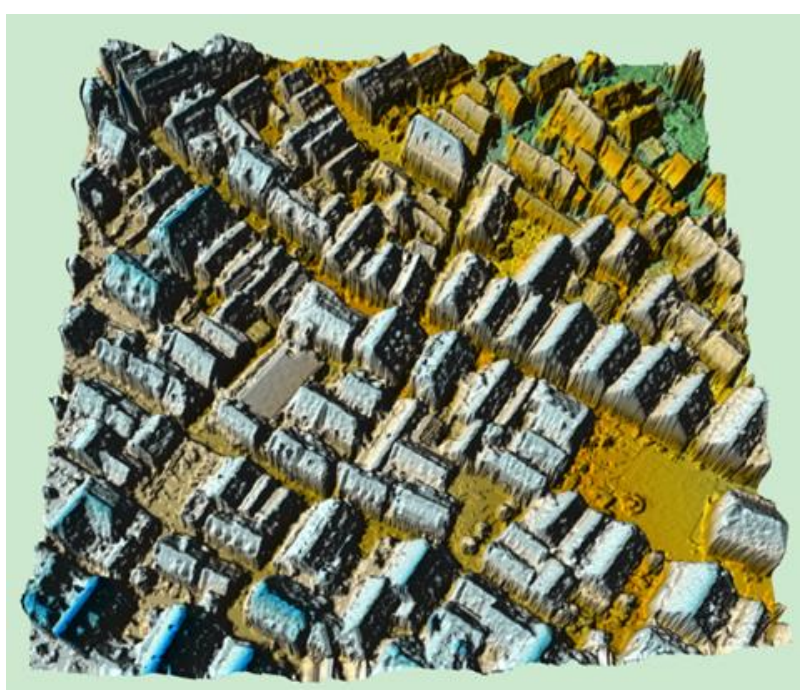

Fig.6 The DSM from the proposed method in Vaihingen

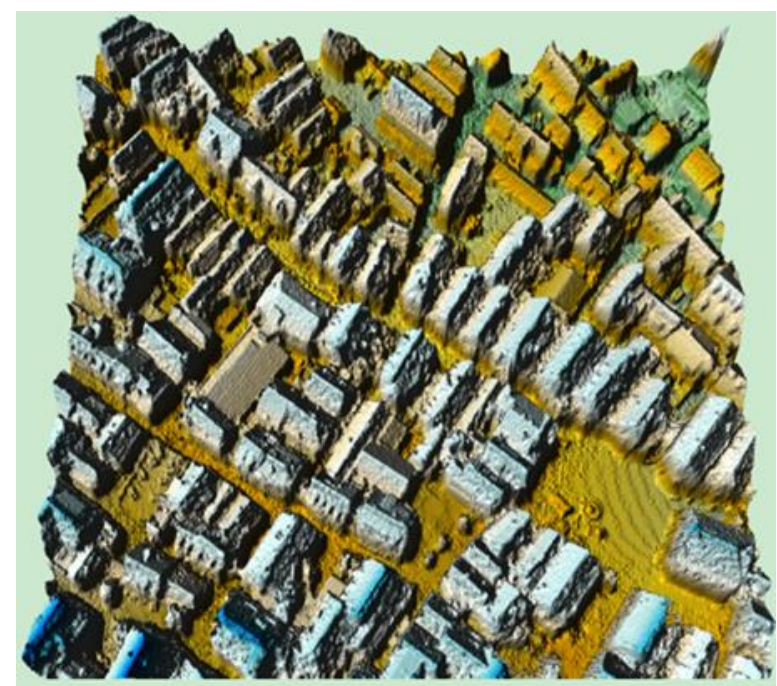

Fig.7 The DSM from the SGM method in Vaihingen

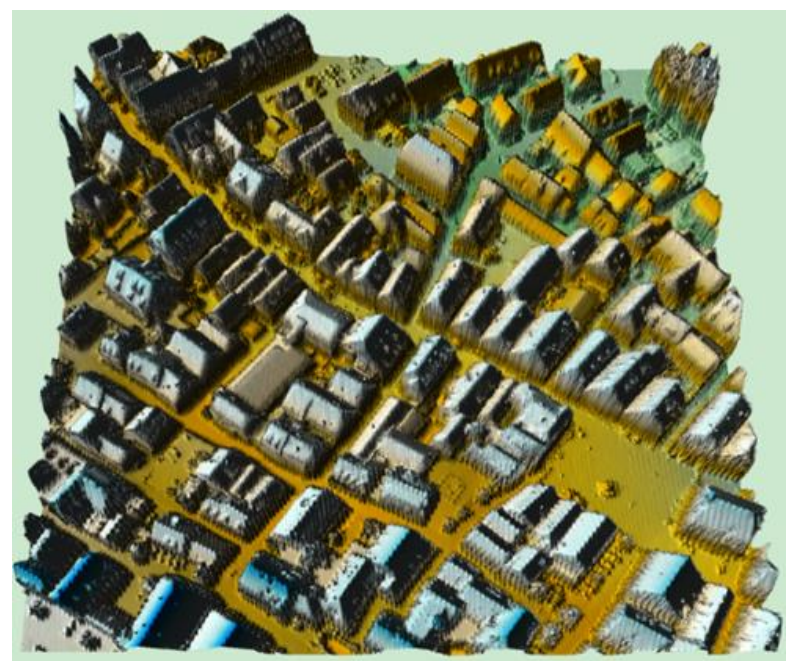

Fig. 8 The DSM from the LiDAR data in Vaihingen

\subsection{Pleiades-1 Images in Hong Kong}

The Pleiades-1 images in Hong Kong were used to evaluate the performance in metropolitan areas like Hong Kong (Fig. 9). The ground resolution is $0.5 \mathrm{~m} / \mathrm{pixel}$ for the Pleiades- 1 images. The reference data used in this experiment is the DSM data derived from airborne laser canning with a spatial resolution of $1 \mathrm{~m}$.

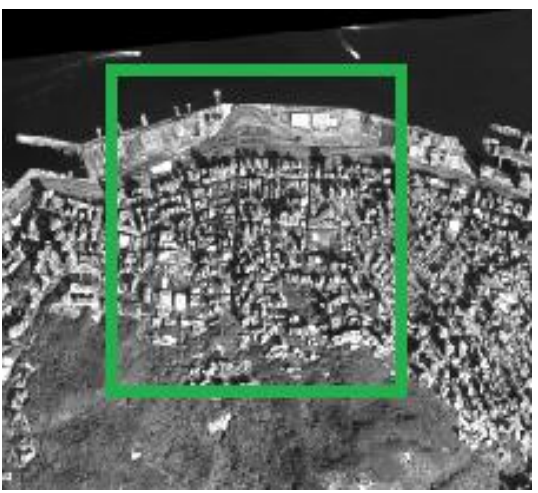

Fig.9 The Pleiades-1 image in Hong Kong.

The statistical evaluation results are shown in Table 2 . In this experiment, all the statistical values show that the proposed method is clearly better than the SGM. This is also supported by the visual inspection from Fig. 10 to Fig. 12. In the flat area in the northern part of the area, the DSM from SGM is smoother than the proposed method, while in the southern part, where buildings are densely located and occlusions are severe, the SGM failed to recover most of the tall buildings. This is mainly because the matching propagation of the proposed method begins by extracting the features, while the SGM use the global optimization based on the smoothness constraint, which may lead to the over-smoothing in the obtained $3 \mathrm{D}$ data.

Table 2 The statistical result of the experiment in Hong Kong

\begin{tabular}{|c|c|c|c|c|}
\hline & $\begin{array}{c}\text { RMSE } \\
(\mathrm{m})\end{array}$ & $\begin{array}{c}\text { StD } \\
(\mathrm{m})\end{array}$ & $\begin{array}{c}\text { Maximum } \\
(\mathrm{m})\end{array}$ & $\begin{array}{c}\text { Density(p } \\
\left.\mathrm{ts} / \mathrm{m}^{2}\right)\end{array}$ \\
\hline $\begin{array}{c}\text { Proposed } \\
\text { Method }\end{array}$ & 5.50 & 5.41 & 39.65 & 2.2 \\
\hline SGM & 6.81 & 6.02 & 80.70 & 2.1 \\
\hline
\end{tabular}




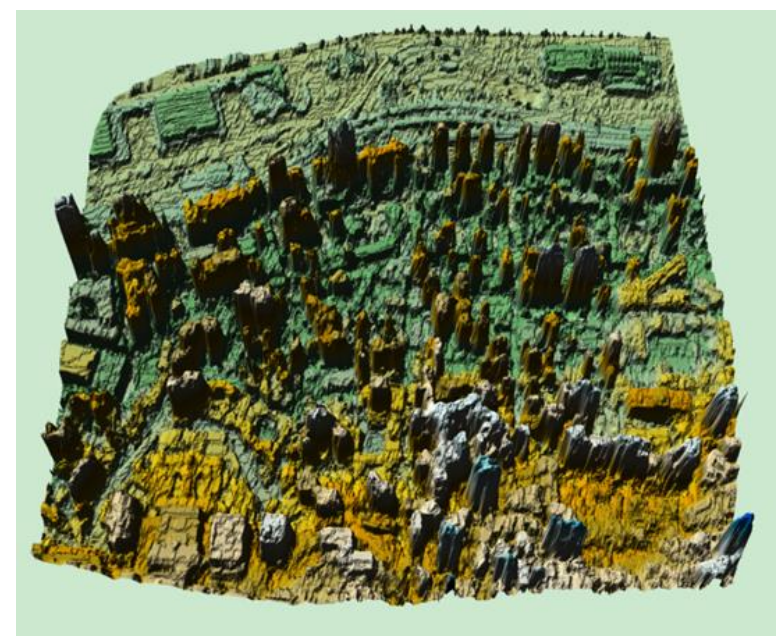

Fig.10 The DSM from the proposed method in Hong Kong

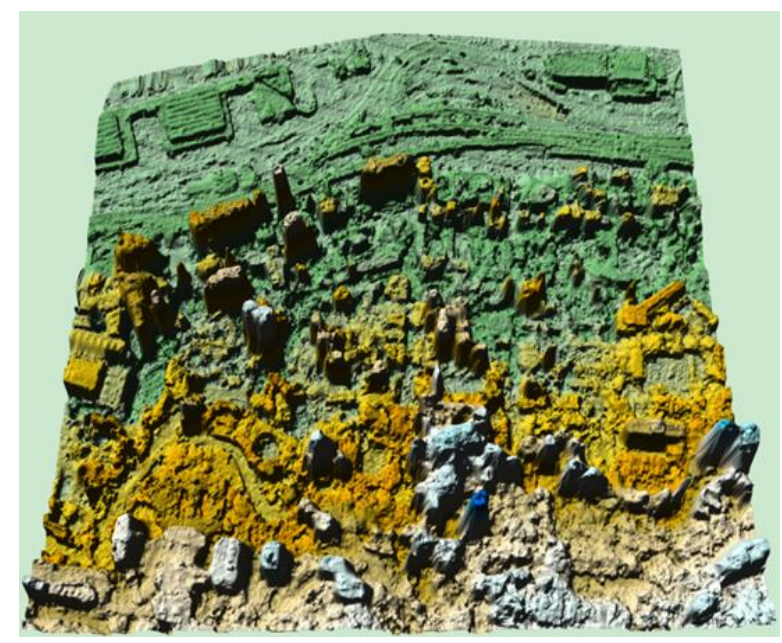

Fig.11 The DSM from the SGM in Hong Kong

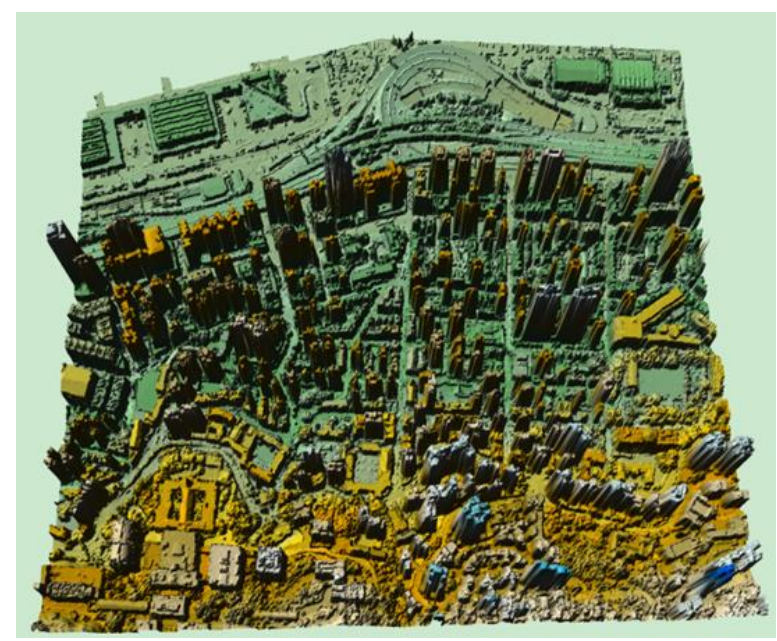

Fig.12 The DSM from LiDAR data in Hong Kong.

\section{CONCLUSIONS AND DISCUSSION}

In this paper, an integrated image matching and segmentation method has been presented for photogrammetric point clouds generation in urban areas. This image matching method is especially designed for solving the problems of urban modelling from high-resolution imagery. Experiment using aerial images in Vaihingen, Germany and high-resolution satellite images in Hong Kong were carried out to evaluate the performance of this approach. As evidenced by visual inspection and quantitative comparisons with airborne laser scanning data and point cloud results from the SGM method, this approach produced dense and reliable result in urban areas, and performed even better than the SGM method in the densely built-up areas. The execution time of the proposed method is comparable to SGM. Besides, the image based point cloud generation can achieve a density of pixel level, which is much higher than the traditional laser scanning data. All of these advantages make the proposed approach very useful for 3D reconstruction in urban areas.

Future work will include the further refinement of the disparity and improvement of the efficiency and the accuracy of the segmentation. Although the proposed method uses the triangulation constraint to narrow the searching range of the candidate match, it is still relatively slower compared with the SGM. Additional work could be done on parallel computing or GPU computing to improve the matching efficiency. Besides, currently segmentation is implemented by ignoring some ambiguous edges to ensure the accuracy, more effort is needed to clarify rather than ignore.

\section{REFERENCE}

Ackermann, F., Krzystek, P., 1991. MATCH-T: Automatic mensuration of digital elevation models, Proceedings of Technical Seminar of the Sociedad Espanola de Cartografia Fotogrammetria y Teledeteccion, pp. 67-73.

Baillard, C., Maître, H., 1999. 3-D Reconstruction of Urban Scenes from Aerial Stereo Imagery: A Focusing Strategy. Computer Vision and Image Understanding 76, 244-258.

Bleyer, M., Gelautz, M., 2005. Graph-based surface reconstruction from stereo pairs using image segmentation, Electronic Imaging 2005. International Society for Optics and Photonics, pp. 288-299.

Bleyer, M., Rhemann, C., Rother, C., 2011a. PatchMatch StereoStereo Matching with Slanted Support Windows, Bmvc, pp. 1-11.

Bleyer, M., Rother, C., Kohli, P., Scharstein, D., Sinha, S., 2011b. Object stereo-joint stereo matching and object segmentation, Computer Vision and Pattern Recognition (CVPR), 2011 IEEE Conference on. IEEE, pp. 30813088 .

Brenner, C., 2005. Building reconstruction from images and laser scanning. International Journal of Applied Earth Observation and Geoinformation 6, 187-198.

Fischler, M.A., Bolles, R.C., 1981. Random sample consensus: a paradigm for model fitting with applications to image analysis and automated cartography. Communications of the ACM 24, 381-395.

Gruen, A., Agouris, P., 1997. Automatic Extraction of ManMade Objects from Aerial and Space Images. Semantic Modeling for the Acquisition of Topographic Information from Images and Maps: SMATI 97, 228.

Habib, A.F., Zhai, R., Kim, C., 2010. Generation of complex polyhedral building models by integrating stereo-aerial imagery and lidar data. Photogrammetric engineering \& remote sensing 76, 609-623.

Helpke, C., 1995. State-of-the-art of digital photogrammetric workstations for topographic applications. Photogrammetric Engineering and Remote Sensing 61, 49-56. 
Hirschmüller, H., 2001. Improvements in real-time correlationbased stereo vision, Stereo and Multi-Baseline Vision, 2001.(SMBV 2001). Proceedings. IEEE Workshop on. IEEE, pp. 141-148.

Hirschmuller, H., 2008. Stereo processing by semiglobal matching and mutual information. IEEE Transactions on pattern analysis and machine intelligence 30, 328-341.

Hirschmuller, H., 2011. Semi-global matching-motivation, developments and applications. Invited Paper at the Photogrammetric Week

Huising, E.J., Gomes Pereira, L.M., 1998. Errors and accuracy estimates of laser data acquired by various laser scanning systems for topographic applications. ISPRS Journal of Photogrammetry and Remote Sensing 53, 245-261.

Klaus, A., Sormann, M., Karner, K., 2006. Segment-based stereo matching using belief propagation and a self-adapting dissimilarity measure, Pattern Recognition, 2006. ICPR 2006. 18th International Conference on. IEEE, pp. 15-18.

Lhuillier, M., Quan, L., 2002. Match propagation for imagebased modeling and rendering. IEEE Transactions on Pattern Analysis and Machine Intelligence 24, 1140-1146.

Li, D., Zhang, G., Wu, Z., Yi, L., 2010. An Edge Embedded Marker-Based Watershed Algorithm for High Spatial Resolution Remote Sensing Image Segmentation. IEEE Transactions on Image Processing 19, 2781-2787.

Marinov, B., 2007. Buildings' modelling for digital orthorectification of urban areas images. Proc. of PIA07Photogrammetric Image Analyses, International Archives of Photogrammetry, Remote Sensing and Spatial Information Sciences (IAPRSSIS) 36, W49B.

Meer, P., Georgescu, B., 2001. Edge detection with embedded confidence. IEEE Transactions on pattern analysis and machine intelligence 23, 1351-1365.

Megyesi, Z., Chetverikov, D., 2004. Affine propagation for surface reconstruction in wide baseline stereo, Pattern Recognition, 2004. ICPR 2004. Proceedings of the 17th International Conference on. IEEE, pp. 76-79.

Musialski, P., Wonka, P., Aliaga, D.G., Wimmer, M., Gool, L.v., Purgathofer, W., 2013. A survey of urban reconstruction, Computer graphics forum. Wiley Online Library, pp. 146-177.

Otto, G.P., Chau, T.K.W., 1989. 'Region-growing' algorithm for matching of terrain images. Image and Vision Computing 7, 83-94.

Paparoditis, N., Maillet, G., Taillandier, F., Jibrini, H., Jung, F., Guigues, L., Boldo, D., 2001. Multi-image 3D feature and DSM extraction for change detection and building reconstruction. Automatic Extraction of Man-Made objects from aerial and space images (III), 217-230.

Scharstein, D., Szeliski, R., 2002. A taxonomy and evaluation of dense two-frame stereo correspondence algorithms. International journal of computer vision 47, 7-42.

Wu, B., Zhang, Y., Zhu, Q., 2011. A triangulation-based hierarchical image matching method for wide-baseline images. Photogrammetric Engineering \& Remote Sensing 77, 695-708.

Wu, B., Zhang, Y., Zhu, Q., 2012. Integrated point and edge matching on poor textural images constrained by selfadaptive triangulations. ISPRS journal of photogrammetry and remote sensing 68, 40-55.

Xu, S., Zhang, F., He, X., Shen, X., Zhang, X., 2015. PM-PM: PatchMatch with Potts model for object segmentation and stereo matching. IEEE Transactions on Image Processing 24, 2182-2196.

Zhang, C., Li, Z., Cheng, Y., Cai, R., Chao, H., Rui, Y., 2015. Meshstereo: A global stereo model with mesh alignment regularization for view interpolation, Proceedings of the
IEEE International Conference on Computer Vision, pp. 2057-2065

Zhang, L., 2005. Automatic digital surfece model(DSM) generation from linear array images. MitteilungenInstitut fur Geodasie und Photogrammetrie an der Eidgenossischen Technischen Hochschule Zurich.

Zhang, L., Gruen, A., 2006. Multi-image matching for DSM generation from IKONOS imagery. ISPRS Journal of Photogrammetry and Remote Sensing 60, 195-211.

Zhu, Q., Zhang, Y., Wu, B., Zhang, Y., 2010. Multiple closerange image matching based on a self-adaptive triangle constraint. The Photogrammetric Record 25, 437-453.

Zhu, Q., Zhao, J., Lin, H., Gong, J., 2005. Triangulation of welldefined points as a constraint for reliable image matching. Photogrammetric Engineering \& Remote Sensing 71, 1063-1069. 